\title{
USO DE AISLAMIENTOS ENDOFÍTICOS DE Trichoderma spp., PARA EL BIOCONTROL DEL MAL DE PANAMÁ (Fusarium oxysporum f. sp. cubense) RAZA 1 EN VITROPLANTAS DE BANANO DEL CULTIVAR GROS MICHEL (AAA) EN CONDICIONES DE INVERNADERO
}

\author{
USE OF ENDOPHYTIC INSULATION OF Trichoderma spp., FOR BIOCONTROL OF PANAMA \\ DESEASE (Fusarium oxysporum f. Sp. Cubense) RACE I, IN VITRO PLANTS OF BANANA, \\ GROS MICHEL VARIETY (AAA) UNDER GREENHOUSE
}

\author{
Álvaro José Caballero Hernández'; Luis Ernesto Pocasangre Enamorado ${ }^{2}$; Fernando Casanoves ${ }^{3}$; Jacques Avelino \\ Ana Cecilia Tapia Fernández ${ }^{5}$, Juan Luis Ortiz \\ ${ }^{1}$ M.Sc. Agricultura Ecológica, Departamento de Agroecología Tropical. Facultad de Ciencia y Tecnología. Universidad \\ Nacional Autónoma de Nicaragua-León \\ ${ }^{2}$ Ph.D. Patología de Plantas, Universidad EARTH, Costa Rica \\ ${ }^{3}$ Ph.D. Ciencias Estadísticas, Unidad de Biometría, CATIE, Turrialba, Costa Rica \\ ${ }^{4}$ Ph.D. Fitopatología, CATIE-CIRADH, Turrialba, Costa Rica \\ ${ }^{5}$ M.Sc. Fitopatología, Sede de Atlántico, UCR, Turrialba, Costa Rica \\ ${ }^{6}$ M.Sc. Recursos Filogenéticos y Biotecnología, Laboratorio de Biotecnología, CATIE, Turrialba, Costa Rica
}

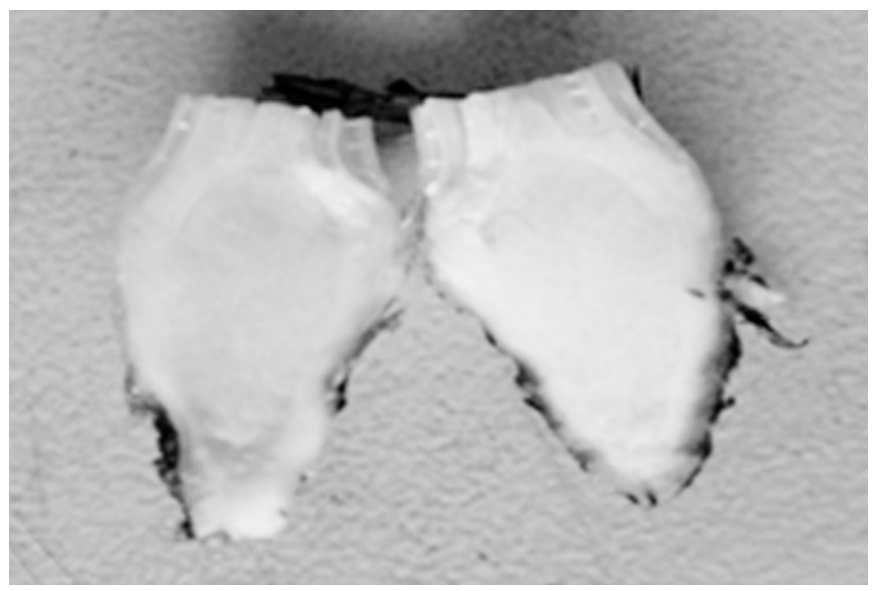

\section{RESUMEN}

El objetivo de la presente investigación fue seleccionar aislamientos endofíticos de Trichoderma spp., para el biocontrol de Fusarium oxysporum $\mathrm{f}$. sp. cubense raza 1 . Se evaluaron los tres aislamientos más patogénicos FOC2, FOC4, FOC8 obtenidos del criobanco del Laboratorio de Fitopatología del CATIE, en una prueba de antibiosis y posteriormente se procedió a realizar la prueba de biocontrol con veinte aislamientos endofíticos de Trichoderma spp. y dos aislamientos FOC2 y FOC4 en vitroplantas de Gros Michel (AAA) en condiciones de invernadero. Por medio de la técnica de cocultivo veinte aislados de Trichoderma spp., inhibieron el crecimiento radial de FOC hasta en un 53,46\%. En el bioensayo de biocontrol, los aislamientos endofíticos de Trichoderma spp., presentaron un mínimo porcentaje de incidencia con $37,5 \%$ del tratamiento TJ5, en comparación al testigo absoluto que no presentó incidencia. Así mismo los tratamientos TC9, TP3 y TCL1 redujeron desde un $92 \%$ hasta $90 \%$ los síntomas externos en comparación a los testigos referenciales. Los síntomas internos del cormo se redujeron hasta un $74 \%$ por el tratamiento TC9. Adicionalmente se detectó que plantas protegidas con los aislamientos endofíticos de Trichoderma spp., promovieron el crecimiento vegetativo de la planta en peso de la raíz y follaje.

Palabras clave: Fusarium oxysporum f. sp. cubense, Mal de Panamá, Gros Michel (AAA), hongos endofíticos, control biológico, Trichoderma spp.

\section{ABSTRACT}

The objective of this research was to select isolates of endophytic fungi of Trichoderma spp., for the biocontrol of Fusarium oxysporum f. sp. cubense race 1 . We evaluated the three most pathogenic isolates FOC2, FOC4, FOC 8 cryobank obtained from the Laboratory of Plant Pathology and Nematology at CATIE, in a test of antibiosis and then proceeded to test twenty biocontrol isolates of Trichoderma spp., and two isolates FOC4 and FOC2 in vitroplants of banana Gros Michel (AAA) under greenhouse conditions. Through the Coculture technique twenty endophytic fungi inhibited radial growth of Foc up to 53, 46\% in comparison with reference control. In the bioassay of Biocontrol, most treatments showed the first symptoms after the third week. Protected plants with endophytic fungi delayed the appereance of the disease until the fourth week. The results showed that endophytic fungi showed a small percentage of incidence being the TJ5 who excelled with the lowest percentage of $37,5 \%$, without showing any symptoms absolute control. Also TC9 endophytic fungi, TP3 and TCL1 reduced from $92 \%$ to $90 \%$ external symptoms, compared to reference control. Corm internal symptoms were reduced to $74 \%$ by the endophytic fungus TC9. Additionally it was found that protected plants endophytic fungi in promoting growth in the variables of foliage weight and root weight. Keywords: Fusarium oxysporum f. sp. cubense, Panama disease, banana, Gros Michel (AAA), endophytic fungus, biological control, Trichoderma spp. 
$\mathrm{E}$ 1 banano Musa spp., se establece en más de 120 países, constituyendo un alimento importante en la dieta básica de 400 millones de personas y una fuente de ingresos económicos para países en desarrollo en África, Asia, Latinoamérica y El Caribe; con una producción de 104 millones de toneladas al año, en aproximadamente 10 millones de ha (Aurore et al., 2009).

Las grandes limitantes de producción son enfermedades como Sigatoka negra causada por Mycosphaerella fijiensis Morelet, el volcamiento de las plantas causados por el fitonematodo Radopholus similes (Cob) Thorne y Mal de Panamá causado por el hongo Fusarium oxysporum f. sp. cubense (Foc) considerada una de las enfermedades fungosa de marchitez vascular más destructivas registradas en la historia bananera (Ploetz 1994; Moore et al., 1995; Pérez et al., 2009)

Los síntomas externos inician con un amarillamiento y marchitez de las hojas adultas, progresando a las hojas jóvenes y causando la muerte total, además de presentar una rajadura en el pseudotallo a nivel del suelo (Ploetz 1994; Ploetz 2006) (figura 1). A nivel interno de la planta la infección avanza mostrando una decoloración del cormo y necrosis en los vasos xilemáticos del pseudotallo (Ploetz 1994; Pocasangre 2008; Pocasangre 2009) (figura 1). Foc produce en el suelo clamidosporas capaces de sobrevivir más de 20 años en ausencia de su hospedero (Ploetz 2006) (figura 1).

Actualmente no existen medidas de combate químico eficientes para la enfermedad (Ploetz 1994), ni buenas prácticas culturales que reduzcan su incidencia y severidad (Ploetz 2006; Pocasangre y Pérez-Vicente 2010). De acuerdo a lo anterior la presente investigación tiene como finalidad evaluar la utilización de aislamientos endofíticos de Trichoderma spp., para el combate biológico del Mal de Panamá causado por Fusarium oxysporum f. sp. cubense raza 1, en vitroplantas del cultivar Gros Michel (AAA), bajo condiciones de invernadero.

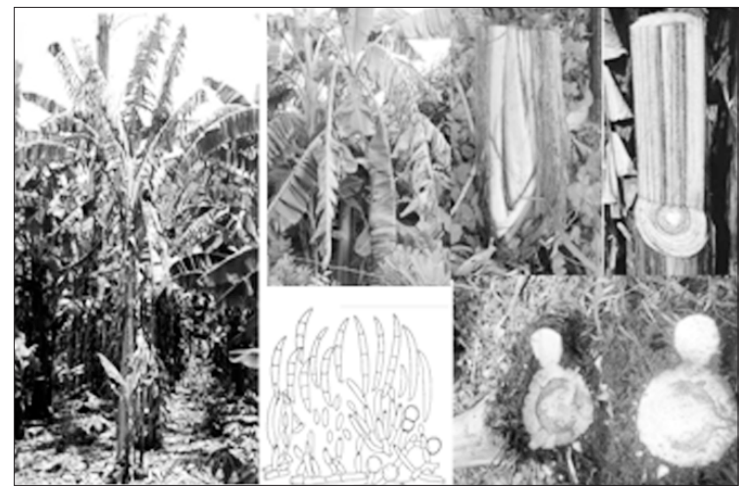

Figura 1. Síntomas externos e internos de la enfermedad Mal de Panamá en plantaciones de banano del cultivar Gros Michel (AAA) y estructuras reproductivas (microconidias, macroconidias y clamidiosporas) del patógeno Fusarium oxysporum f. sp. cubense raza 1

\section{MATERIAL Y MÉTODOS}

Ubicación geográfica de la investigación. Este estudio se realizó en el laboratorio de Nematología y Fitopatología del Centro Agronómico Tropical de Investigación y Enseñanza (CATIE), ubicado en el cantón de Turrialba, provincia de Cartago, Costa Rica.

\section{Material experimental}

Aislamientos de Fusarium oxysporum f. sp. Cubense. Se utilizaron tres aislamientos purificados de Fusarium oxysporum f. sp. cubense (Foc) raza 1, para el bioensayo de antibiosis y dos aislamientos para el bioensayo biocontrol. Los aislamientos son FOC2, FOC4 Y FOC8 (Lara, 2009), obtenidos del Criobanco del Laboratorio de Nematología y Fitopatología del CATIE, Turrialba, Costa Rica.

Aislamientos endofíticos de Trichoderma spp. Se utilizaron 50 aislamiento endofíticos del género Trichoderma spp., que fueron aislados de los cultivares Cavendish (Subgrupo, AAA), como agentes de biocontrol de fitonematodos en pruebas in vitro e in vivo realizadas en el Laboratorio de Nematología y Fitopatología del CATIE (Cañizares 2003; Menjivar 2005).

Material Vegetal. Se utilizaron plantas de banano del cultivar Gros Michel (AAA) con once semanas de endurecimiento colectivo en invernadero, provenientes del área de cultivos de tejidos del Laboratorio de Biotecnología del CATIE.

Cultivo y multiplicación de los aislamientos de Fusarium oxysporum f. sp. cubense y aislamientos endofíticos de Trichoderma spp. Se cultivaron colonias puras de los tres aislamientos de Foc y de los 50 aislamientos endofíticos de Trichoderma spp., en Papa-Dextrosa-Agar (PDA) al 100\% con hidróxido de potasio al 85\%. Estos aislamientos fueron almacenados a $24^{\circ} \mathrm{C}$ por dos semanas en una incubadora, para el crecimiento y esporulación de las colonias. Después se multiplicaron los aislamientos endofíticos de Trichoderma spp., y Foc, extrayéndose discos de PDA al 100\% de $5 \mathrm{~mm}$ de diámetro que contenían micelio y estructura reproductivas (esporas) para ser trasladadas a nuevos platos Petri con PDA al $100 \%$ en condiciones asépticas. Los platos sembrados con los aislamientos fueron almacenados a $24^{\circ} \mathrm{C}$ por dos semanas en una incubadora.

Suspensión de esporas de Fusarium oxysporum f. sp. cubense y Trichoderma spp. Cultivos puros de los aislamientos de Fusarium oxysporum f. sp. cubense y de Trichoderma spp., crecidos en PDA al 100\% y almacenados a $24^{\circ} \mathrm{C}$ durante dos semanas en una incubadora, fueron utilizados para la obtención de una suspensión de esporas.

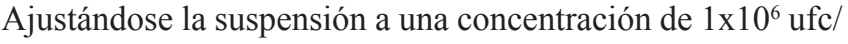
$\mathrm{ml}$. 
Bioensayo 1. Prueba de antibiosis a través de la técnica de cocultivo sobre los tres aislamientos de Fusarium oxysporum f. sp. cubense y endofíticos de Trichoderma spp. Un disco de PDA de $5 \mathrm{~mm}$ de diámetro de Foc fue colocado al extremo de un plato Petri que contenía PDA al $100 \%$. Simultáneamente al extremo opuesto se colocó un disco de PDA de $5 \mathrm{~mm}$ de diámetro de los aislamientos endofíticos de Trichoderma spp. Cada plato fue almacenado a $24^{\circ} \mathrm{C}$ por 8 días. Se establecieron tres testigos referenciales que correspondieron al cultivo de los tres aislamientos de Foc sin los discos opuestos de Trichoderma spp. Se realizaron tres repeticiones por cada tratamiento. El crecimiento de las colonias de Foc y de Trichoderma spp., fue evaluado cada 24 horas durante un periodo de ocho días, con el uso de una regla graduada en centímetros. En cada plato Petri se midió el crecimiento radial de las colonias de los hongos hacia el lado izquierdo y derecho del centro del disco que las contenía.

Bioensayo 2. Prueba de biocontrol. Para la prueba de biocontrol se seleccionaron 20 aislamientos endofíticos de Trichoderma spp., que presentaron el mayor porcentaje de inhibición del crecimiento radical de Foc. La prueba consistió en la evaluación de la incidencia de la marchitez y la severidad de los síntomas externos e internos de la enfermedad Mal de Panamá en vitroplantas de banano del cultivar Gros Michel (AAA), empleando la escala de Orjeda (1998).

Las vitroplantas fueron protegidas con los aislamientos endofíticos de Trichoderma spp., sumergiendo las raíces en una suspensión conidial ajustada de $1 \times 10^{6} \mathrm{ufc} / \mathrm{ml}$ durante 15 minutos. Estas fueron trasplantadas en contenedores plásticos de $250 \mathrm{~cm}^{3}$ de capacidad que contenían una mezcla esterilizada de tierra y arena en una relación 1:1 y se realizaron ocho repeticiones por cada tratamiento, estas unidades experimentales se distribuyeron al azar en ocho mesas en el invernadero de musáceas donde permanecieron 11 semanas a una temperatura $27.3 \pm 2^{\circ} \mathrm{C}$. Tres semanas después se realizó la inoculación con los aislamientos 2 y 4 de Fusarium oxysporum f. $\mathrm{sp}$. cubense, aplicando cinco $\mathrm{ml}$ de una suspensión ajustada de Foc a una concentración $1 \times 10^{6} \mathrm{ufc} /$ $\mathrm{ml}$ en tres agujeros de uno a dos $\mathrm{cm}$ de profundidad realizado en el sustrato alrededor del área radical y cercana a la base del rizoma.

Cuatro semanas después se realizó una segunda inoculación de Foc siguiendo el mismo procedimiento.
Se establecieron dos testigos referenciales con inoculación de los dos aislamientos dos y cuatro de Foc, sin previa protección de las inoculaciones de los aislamientos endofíticos de Trichoderma spp., además, se estableció un testigo absoluto.

Métodos estadísticos. Los datos fueron analizados mediante el programa estadísticos InfoStat usando una interfase de la plataforma R (Di Rienzo et al., 2009). Realizándose análisis de varianza (ANDEVA) para efectos fijos, bajo la óptica de los modelos lineales generales y mixtos, para las variables que se evaluaron en cada uno de los dos bioensayos. Se usaron modelos lineales generales y mixtos cuando las variables no presentaron varianzas homogéneas entre los tratamientos y se realizó una comparación de medias de los tratamientos con LSD Fisher para la severidad de los síntomas externos e internos de la enfermedad.

\section{RESULTADOS Y DISCUSIÓN}

Bioensayo 1. Prueba de antibiosis sobre los tres aislamientos de Fusarium oxysporum f. sp. cubense. El hongo endofítico TB1 inhibió el 53,46\% del crecimiento radial del aislamiento cuatro de Foc, en comparación al testigo referencial FOC4 que creció el $100 \%$ con 2,17 centímetros. De igual manera TV3 inhibió el 38,82\%; TV4 con 38,16\% y TCL4 con $36,85 \%$ del crecimiento radial del aislamiento dos de Foc, en comparación del testigo referencial FOC2 que creció el $100 \%$ con 1,52 centímetros. Siendo éstos aislamiento de Trichoderma spp., que obtuvieron el mayor porcentaje de inhibición de crecimiento radial para los aislados FOC2 y FOC4 en la prueba de cocultivo. El aislado FOC8 presentó un menor crecimiento radial. Por lo que se procedió a tomar los aislados FOC2 y FOC4 para evaluaciones de biocontrol (figura 2 y 3 ).

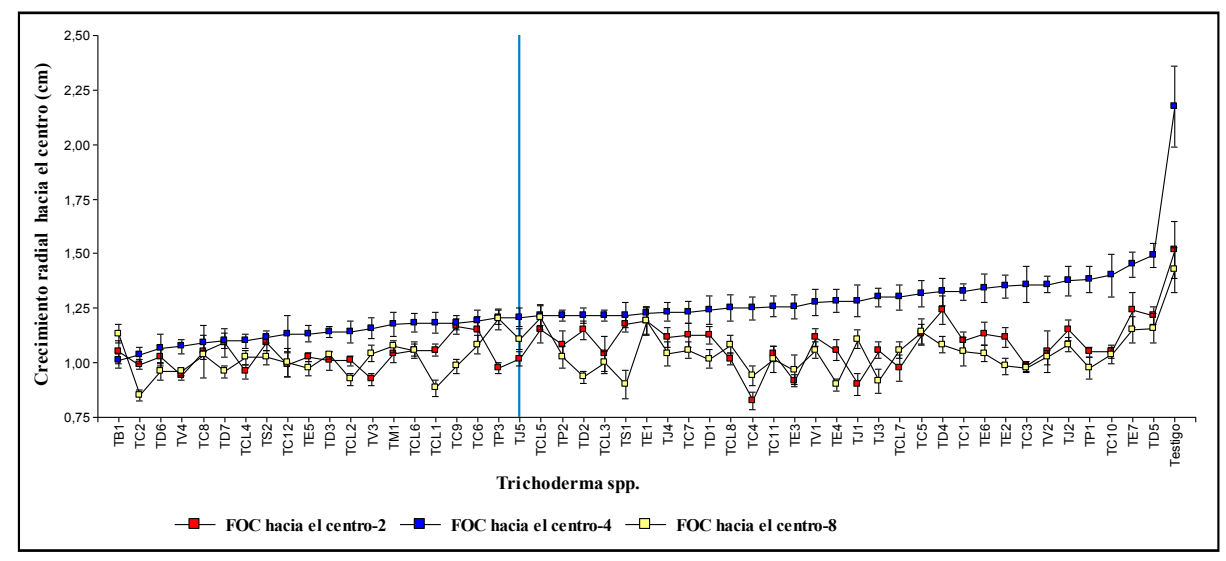

Figura 2. Efecto de hongos endofíticos sobre el crecimiento de Fusarium oxysporum f. sp. cubense en ocho evaluaciones. 


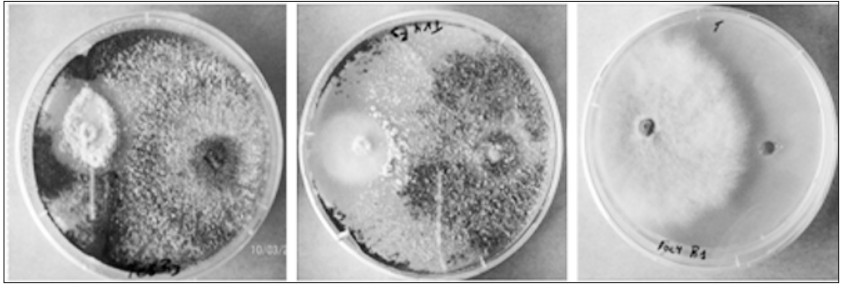

Figura 3. Inhibición del crecimiento radial en prueba de cocultivo de los aislamientos endofíticos de Trichoderma spp., sobre Fusarium oxysporum $\mathrm{f}$. sp. cubense raza 1 al cabo de la octava medición.
Pero la incidencia de la enfermedad se empezó a registrar a partir de la tercera semana después de las inoculaciones con los aislados dos y cuatro de Foc para la mayoría de los tratamientos, con los primeros síntomas externos como el amarillamiento en las hojas adultas. El testigo absoluto no presentó síntomas de la enfermedad, debido a que estas no fueron inoculadas con los hongos endofíticos ni con los aislamientos de FOC2 y FOC4 (figura 4).

En la prueba de cocultivo, el efecto de reducción del aislamiento cuatro de Foc es mayor al confrontarse con los aislados endofíticos de Trichoderma spp., debido a que el crecimiento del micelio de Trichoderma spp., compite por espacio y fuentes nutricionales que están presentes en el plato Petri de PDA al $100 \%$, generando un efecto indirecto en la

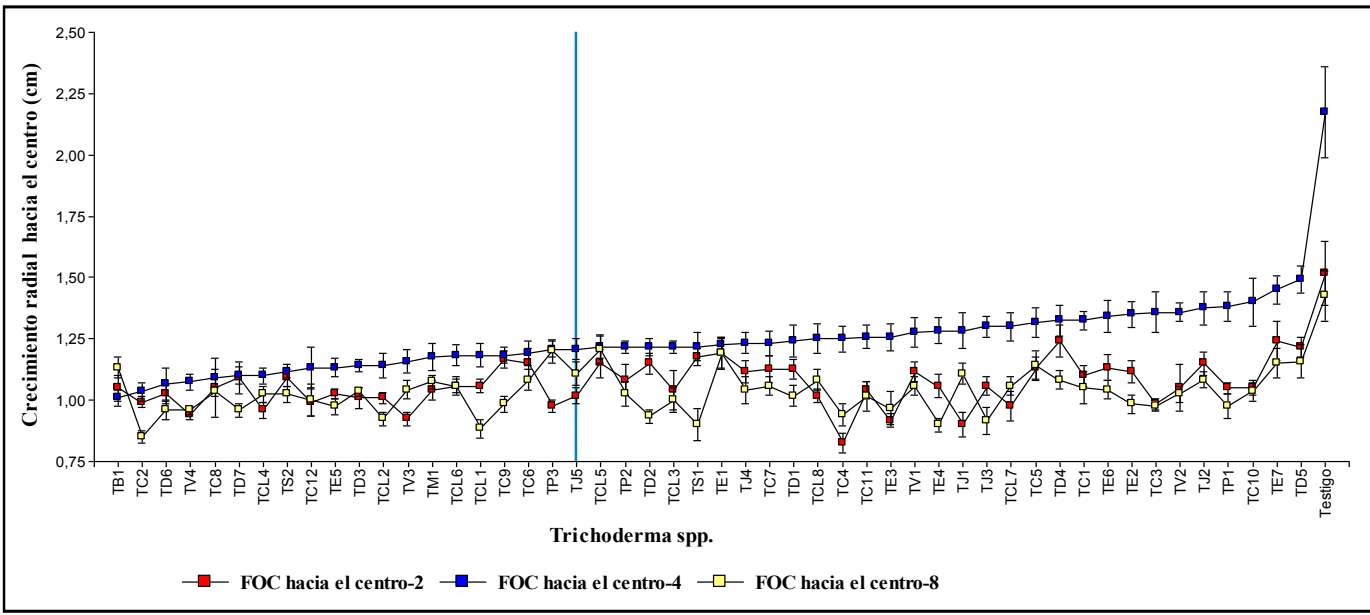

Figura 4. Incidencia del Mal de Panamá con dos aislamientos de Fusarium oxysporum f. sp. cubense en vitroplantas de banano del cultivar Gros Michel (AAA) durante ochos semanas de evaluación. reducción del crecimiento radial del aislado por ocupar su espacio y extraer los suministro de nutrientes (Howell 2003; Woo et al., 2006; Vinale et al., 2008). Al seleccionar los 20 aislamientos endofíticos de Trichoderma spp., para someterlos al segundo bioensayo, estos no presentaron consistencia en la prueba de biocontrol, descartando el mecanismo de antibiosis por no generarse en esta prueba. Lara (2009) no encontró consistencia en la prueba de biocontrol, al utilizar aislamientos de bacterias endofíticas, seleccionadas por la prueba de cocultivo. Asimismo Noreskal (2009) evaluó aislamiento de hongos y bacterias endofíticas para la prueba de biocontrol, presentando un comportamiento distinto en la prueba de biocontrol para la severidad de los síntomas externos e internos de la enfermedad Mal de Panamá.

Bioensayo 2. Prueba de biocontrol sobre los dos aislamientos de Fusarium oxysporum $f$. sp. cubense Incidencia y severidad de la enfermedad. Los tratamientos que fueron inoculados previamente con los aislamientos endofíticos de Trichoderma spp., retardaron la aparición de los síntomas externos hasta la cuarta semana de la enfermedad Mal de Panamá en las vitroplantas de banano del cultivar Gros Michel (AAA) en comparación de los testigos referenciales.
Con respecto al tratamiento que presentó el menor porcentaje de incidencia es TJ5-FOC2, en comparación con el resto de las interacciones y los testigos referenciales. El hongo endofítico TJ5 funcionó para los aislamientos FOC2 y FOC4 encontrando un porcentaje de incidencia de 37,5\% y $62,5 \%$ (tabla 1 ).

Tabla 1. Efecto de los aislamientos endofíticos de Trichoderma spp., sobre la incidencia de la enfermedad Mal de Panamá en ochos semanas de evaluación

\begin{tabular}{|c|c|c|c|c|}
\hline \multicolumn{5}{|c|}{ \% Incidencia } \\
\hline \multirow{2}{*}{$\begin{array}{l}\text { Trichoderma } \\
\text { T. abs }\end{array}$} & \multicolumn{2}{|c|}{ FOC2 } & \multicolumn{2}{|c|}{ FOC4 } \\
\hline & & O & $\mathrm{a}$ & \\
\hline TJ5 & 37,5 & $a b$ & 62,5 & bc \\
\hline TV3 & 87,5 & $\mathrm{c}$ & 62,5 & bc \\
\hline TD6 & 87,5 & $\mathrm{c}$ & 62,5 & bc \\
\hline TCL1 & 87,5 & $\mathrm{c}$ & 62,5 & bc \\
\hline TC6 & 62,5 & bc & 87,5 & $\mathrm{c}$ \\
\hline $\mathrm{TC} 2$ & 62,5 & bc & 100 & $\mathrm{c}$ \\
\hline ТВ1 & 75 & bc & 62,5 & bc \\
\hline TS2 & 75 & bc & 100 & $\mathrm{c}$ \\
\hline TP3 & 87,5 & $\mathrm{c}$ & 75 & bc \\
\hline TE5 & 87,5 & $\mathrm{c}$ & 75 & bc \\
\hline TCL6 & 75 & bc & 100 & $\mathrm{c}$ \\
\hline TCL4 & 75 & bc & 87,5 & $\mathrm{c}$ \\
\hline TV4 & 100 & $\mathrm{c}$ & 87,5 & $\mathrm{c}$ \\
\hline TM1 & 87,5 & $\mathrm{c}$ & 100 & $\mathrm{c}$ \\
\hline T. ref. & 87,5 & c & 87,5 & c \\
\hline TD7 & 87,5 & $\mathrm{c}$ & 100 & c \\
\hline TD3 & 100 & c & 87,5 & c \\
\hline TCL2 & 100 & $\mathrm{c}$ & 87,5 & $\mathrm{c}$ \\
\hline TC9 & 87,5 & c & 100 & $\mathrm{c}$ \\
\hline TC8 & 87,5 & c & 87,5 & c \\
\hline TC12 & 100 & c & 100 & c \\
\hline
\end{tabular}


El aislamiento endofítico de Trichoderma spp., TJ5 demostró efecto de reducción en la incidencia al final de la octava medición, estos resultados son consistentes en estudios realizados por Thangavelu et al., (2003) y Mitov y Oliva (1975) donde se presentan reducciones de la enfermedad desde un 48 hasta 51\%. El aislado TJ5 retrasó la aparición de los síntomas externos en más de tres semanas, aumentando el período de incubación de la enfermedad en condiciones de invernadero. Este efecto en retardar el período de incubación de la enfermedad, posiblemente se deba a la colonización y establecimiento temprano en el sustrato, en el sistema radical, en los tejidos internos del cormo y los tejidos vasculares de la planta. Mostrando una mayor competencia para ocupar estos espacios, que son colonizados por la mayoría de los patógenos oportunistas o fitopatógenos.

Con respecto a la severidad el tratamiento que menor amarillamiento y marchitez presentó fue TC9-FOC2, en comparación a los testigos referenciales y el testigo absoluto que no presentó síntomas externos de severidad. Obteniendo TC9 una reducción de la enfermedad del 92\% en el amarillamiento y marchitez para el aislado FOC2, en comparación a los testigos referenciales. Además TP3 y TCL1 redujeron los síntomas externos hasta un $90 \%$ para FOC4 y FOC2 respectivamente (tabla 3 )

Tabla 2. Efecto de los aislamientos endofíticos de Trichoderma spp., y porcentajes de reducción (entre paréntesis) sobre el amarillamiento y severidad de la enfermedad Mal de Panamá en ochos semanas de evaluación

\begin{tabular}{|c|c|c|c|c|}
\hline & \multicolumn{2}{|c|}{ Amarillamiento } & \multicolumn{2}{|l|}{ Marchitez } \\
\hline Trichoderma & FOC2 & FOC4 & FOC2 & FOC4 \\
\hline T.abs. & \multicolumn{2}{|c|}{$1(100)^{\mathrm{a}}$} & \multicolumn{2}{|c|}{$1(100)^{\mathrm{a}}$} \\
\hline TC9 & $1,4(92)^{\mathrm{ab}}$ & $1,73(85)^{\text {bcdef }}$ & $1,4(92)^{\mathrm{ab}}$ & $1,71(86)^{\text {bcdefgh }}$ \\
\hline TP3 & $2,58(68)^{\mathrm{jklmn}}$ & $1,48(90)^{\mathrm{abc}}$ & $2,54(69)^{\operatorname{lmnopq}}$ & $1,45(91)^{\mathrm{abc}}$ \\
\hline TCL1 & $1,52(90)^{\mathrm{abc}}$ & $1,9(82)^{\text {bcdefgh }}$ & $1,52(90)^{\mathrm{abcd}}$ & $1,86(83)^{\text {bcdefghij }}$ \\
\hline $\mathrm{TC} 2$ & $1,6(88)^{\mathrm{bcd}}$ & $2,69(66)^{\mathrm{klmno}}$ & $1,58(88)^{\text {bcde }}$ & $2.65(67)^{\text {nopq }}$ \\
\hline TV3 & $2,57(69)^{\mathrm{jklmn}}$ & $1,63(87)^{\text {bcde }}$ & $2,57(69)^{\mathrm{mnopq}}$ & $1,58(88)^{\text {bcde }}$ \\
\hline TD6 & $1,67(87)^{\text {bcdef }}$ & $1,74(85)^{\text {bcdef }}$ & $1,62(88)^{\text {bcdef }}$ & $1,74(85)^{\text {bcdefghi }}$ \\
\hline TV4 & $1,69(86)^{\text {bcdef }}$ & $3,05(59)^{\text {nop }}$ & $1,69(86)^{\text {bcdefg }}$ & $3,05(59)^{\mathrm{qrs}}$ \\
\hline TS2 & $1,71(86)^{\text {bcdef }}$ & $3,13(57)^{\mathrm{op}}$ & $1,69(86)^{\text {bcdefgh }}$ & $3,04(59)^{\mathrm{qrs}}$ \\
\hline TD3 & $1,73(85)^{\text {bcdef }}$ & $2,13(77)^{\text {defghij }}$ & $1,71(86)^{\text {bcdefgh }}$ & $2,12(78)^{\text {fghijklmn }}$ \\
\hline TB1 & $1,75(85)^{\text {bcdef }}$ & $3,31(54)^{\mathrm{p}}$ & $1,73(85)^{\text {bcdefghi }}$ & $3,22(56)^{\mathrm{rs}}$ \\
\hline TJ5 & $1,76(85)^{\text {bcdef }}$ & $2,13(77)^{\operatorname{defghij}}$ & $1,74(85)^{\text {bcdefghi }}$ & $2,06(79)^{\text {efghijklm }}$ \\
\hline TC8 & $1,81(84)^{\text {bcdefg }}$ & $2,71(66)^{\operatorname{lmno}}$ & $1,77(85)^{\text {bcdefghi }}$ & $2,67(67)^{\text {opqr }}$ \\
\hline TC6 & $1,98(80)^{\text {cdefghi }}$ & $2,46(71)^{\mathrm{ijklm}}$ & $1,98(80)^{\text {cdefghijk }}$ & $2,46(71)^{\mathrm{klmnop}}$ \\
\hline T. ref. & $2,07(79)^{\text {defghij }}$ & $3,35(53)^{\mathrm{p}}$ & $2,02(80)^{\text {defghijklm }}$ & $3,25(55)^{\mathrm{s}}$ \\
\hline $\mathrm{TC} 12$ & $2,21(78)^{\operatorname{defghij}}$ & $2,54(69)^{\mathrm{j} k \mathrm{lmn}}$ & $2,02(80)^{\text {defghijkl }}$ & $2,48(70)^{\mathrm{klmnop}}$ \\
\hline TM1 & $2,13(77)^{\text {defghij }}$ & $2,38(72)^{\mathrm{hijk} k m}$ & $2,04(79)^{\text {defghijklm }}$ & $2,15(77)^{\text {fghijklmno }}$ \\
\hline TCL4 & $2,31(74)^{\mathrm{ghijkl}}$ & $2,15(77)^{\text {efghij }}$ & $2,25(75)^{\mathrm{ijk} k \operatorname{lnno}}$ & $2,1(78)^{\text {efghijklm }}$ \\
\hline TD7 & $2,17(77)^{\text {fghijk }}$ & $2,17(77)^{\mathrm{fghijk}}$ & $2,17(77)^{\text {ghijklmno }}$ & $2,17(77)^{\text {ghijklmno }}$ \\
\hline TCL2 & $3,25(55)^{\mathrm{p}}$ & $2,38(72)^{\mathrm{hijklm}}$ & $3,04(59)^{\mathrm{qrs}}$ & $2,23(75)^{\mathrm{hijk} k m n o}$ \\
\hline TCL6 & $2,4(72)^{\mathrm{hijklm}}$ & $2,58(68)^{\mathrm{j} \mathrm{klmn}}$ & $2,4(72)^{\mathrm{jklmnop}}$ & $2,52(70)^{\operatorname{lmnopq}}$ \\
\hline TE5 & $2,9(62)^{\mathrm{mnop}}$ & $2,83(63)^{\operatorname{lmnop}}$ & $2,93(61)^{\mathrm{pqrs}}$ & $2,81(64)^{\mathrm{pqrs}}$ \\
\hline
\end{tabular}

Datos corresponden al promedio de seis evaluaciones. Letras distintas indican diferencias significativas $(\mathrm{p}<=0.05)$.
Evaluación de síntomas internos. Para la decoloración del cormo los aislamientos endofíticos de Trichoderma spp., TC9, TD3, TD6, TC12, TCL1, TC8, TJ5, TC2, TCL4, TP3, TD7, TC6 y TS2 (figura 3) presentaron los menores niveles de decoloración, en comparación al resto de los tratamientos (figura 4). Sobresaliendo TC9 con 74\% en la reducción de la decoloración del cormo (tabla 4).

Tabla 3. Efecto de los aislamientos endofíticos de Trichoderma spp., y porcentajes de reducción (entre paréntesis) sobre la decoloración del cormo de vitroplantas del banano del cultivar Gros Michel (AAA) en la prueba de biocontrol

\begin{tabular}{ll}
\hline Trichoderma & \multicolumn{1}{c}{ Decoloración del cormo } \\
\hline TC9 & $2,31(74)^{\mathrm{a}}$ \\
TD3 & $2,56(69)^{\mathrm{ab}}$ \\
TD6 & $2,64(67)^{\mathrm{abc}}$ \\
TC12 & $2,7(66)^{\mathrm{abc}}$ \\
TCL1 & $2,71(66)^{\mathrm{abc}}$ \\
TC8 & $2,75(65)^{\mathrm{abc}}$ \\
TJ5 & $2,87(63)^{\mathrm{abcd}}$ \\
TC2 & $2,88(62)^{\mathrm{abcde}}$ \\
TCL4 & $2,94(61)^{\mathrm{abcde}}$ \\
TP3 & $2,95(61)^{\mathrm{abcde}}$ \\
TD7 & $3,16(57)^{\mathrm{abcde}}$ \\
TC6 & $3,25(55)^{\mathrm{abcde}}$ \\
TS2 & $3,36(53)^{\mathrm{abcde}}$ \\
TV4 & $3,36(53)^{\mathrm{bcde}}$ \\
TCL6 & $3,39(52)^{\mathrm{bcde}}$ \\
TB1 & $3,48(50)^{\mathrm{cde}}$ \\
TV3 & $3,55(49)^{\mathrm{cde}}$ \\
TCL2 & $3,57(49)^{\mathrm{cde}}$ \\
TM1 & $3,75(45)^{\mathrm{de}}$ \\
TE5 & $3,93(41)^{\mathrm{e}}$ \\
\hline
\end{tabular}

Letras distintas indican diferencias significativas $(\mathrm{p}<=0.05)$. Las letras son raíz cuadrada.

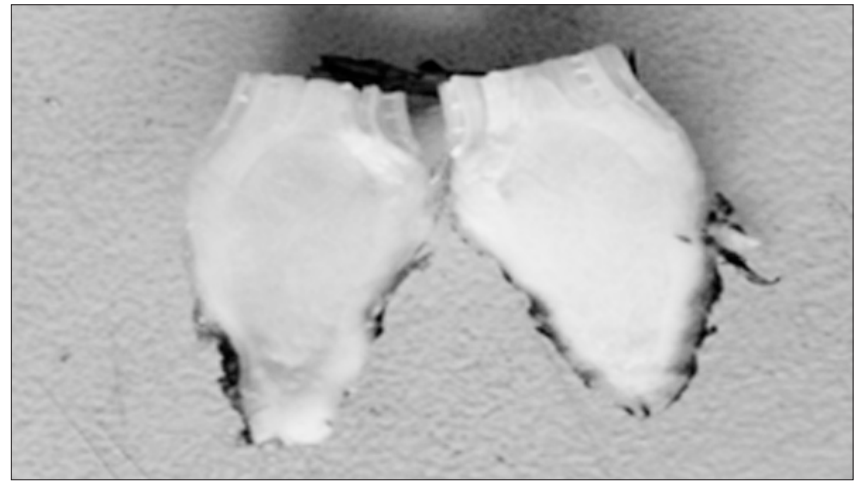

Figura 4. Cormos de plántulas de banano del cultivar Gros Michel (AAA) protegidas con aislamientos endofíticos de Trichoderma spp., sin síntomas de decoloración de la enfermedad Mal de Panamá. 


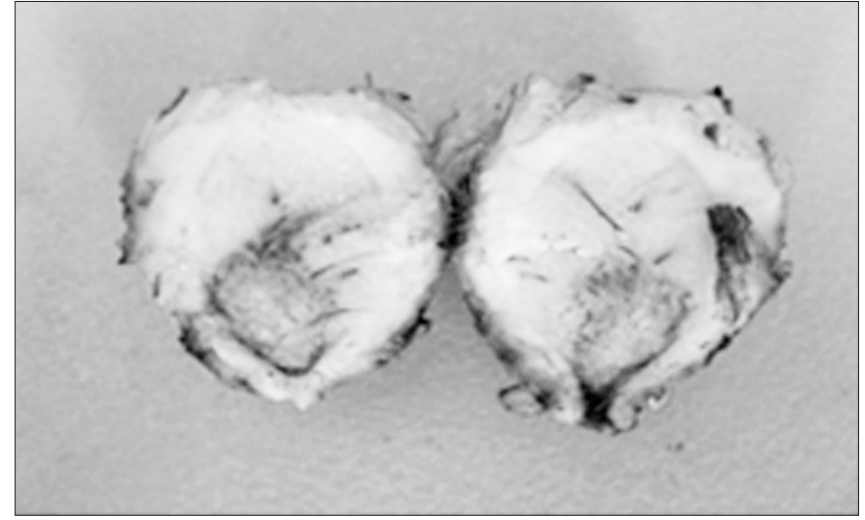

Figura 5. Decoloración severa del cormo en plántulas de banano del cultivar Gros Michel (AAA) causado por la enfermedad Mal de Panamá.

La severidad de los síntomas externos e internos de la enfermedad Mal de Panamá fueron reducidos por las inoculaciones tempranas de los aislamientos endofíticos de Trichoderma spp., en condiciones de invernadero (Figura 4 y 5). Sin embargo, su capacidad de reducción de la severidad difiere con respecto a cada aislado de FOC2 y FOC4 (figura 4 y 5). Estos resultados concuerdan con Pérez et al., (2003) y Pérez et al., (2009) que reportaron la eficacia de Trichoderma harzianum A34 en la reducción de la incidencia y síntomas externos de la enfermedad Mal de Panamá en plantas de Burro CEMSA Bluggoe-ABB, FHIA 03-AABB y FHIA 23-AAAB, además de mantener su producción por más de cincos años en suelos conducibles a la enfermedad.

Medición de las variables peso foliar, peso radical y peso de la planta. Los aislamientos endofíticos de Trichoderma spp., favorecieron un aumento significativo en los parámetros evaluados en las plantas de banano del cultivar de Gros Michel (AAA), siendo TCL1 el que obtuvo el mayor peso de raíz con 9,6 gramos, un peso del follaje de 21,5 gramos y peso de planta de 31 gramos; en comparación al testigo absoluto y los testigos referenciales. El hongo endofítico TCL1 incremento los porcentajes de peso desde 109\% hasta $148 \%$ con respecto al testigo absoluto. El Trichoderma TCL1 tiene un efecto significativo sobre el aislado dos de Fusarium oxysporum f. sp. cubense raza 1(tabla 4).
Tabla 4. Mejores aislamientos de Trichoderma spp., y porcentajes relativos (entre paréntesis) para las variables peso radical $(\mathrm{g})$, peso del follaje $(\mathrm{g})$ y peso de planta $(\mathrm{g})$ al sacrificar las plantas en la finalización del bioensayo de biocontrol

\begin{tabular}{|c|c|c|c|c|c|c|}
\hline \multicolumn{3}{|c|}{ Peso radical } & \multicolumn{2}{|c|}{ Peso del follaje } & \multicolumn{2}{|c|}{ Peso de la planta } \\
\hline Trichoderma & FOC2 & FOC4 & FOC2 & FOC4 & FOC2 & FOC4 \\
\hline TCL1 & $9,6(148)^{\mathrm{a}}$ & $7,1(109)^{\text {bcde }}$ & $21,5(117)^{\mathrm{a}}$ & $17,1(93)^{\text {abcdef }}$ & $31(125)^{\mathrm{a}}$ & $24,2(98)^{\text {abcde }}$ \\
\hline TC9 & $8,6(132)^{\mathrm{ab}}$ & $8,2(126)^{\mathrm{abc}}$ & $18,5(101)^{\text {abcdef }}$ & $19,8(108)^{\mathrm{abc}}$ & $27,1(109)^{\mathrm{abc}}$ & $28(113)^{\mathrm{abc}}$ \\
\hline $\mathrm{TC} 12$ & $8,3(128)^{\mathrm{abc}}$ & $6,4(98)^{\text {bcde }}$ & $19,1(104)^{\text {abcdef }}$ & $16,4(90)^{\text {abcdef }}$ & $27,4(110)^{\mathrm{abc}}$ & $22,8(92)^{\text {bcde }}$ \\
\hline TC8 & $8,1(125)^{\text {abcde }}$ & $6,2(95)^{\text {cde }}$ & $18,5(101)^{\text {abcdef }}$ & $15(82)^{\text {bcdef }}$ & $26,6(107)^{\text {abcd }}$ & $21,2(85)^{\text {bcde }}$ \\
\hline TS2 & $8(123)^{\text {abcde }}$ & $5,1(78)^{e}$ & $20,6(113)^{\mathrm{ab}}$ & $12,1(66)^{\mathrm{f}}$ & $28,6(115)^{\mathrm{ab}}$ & $17,1(69)^{\mathrm{e}}$ \\
\hline TD7 & $6,6(102)^{\text {bde }}$ & $7,8(120)^{\text {abcde }}$ & $19,3(105)^{\text {abcde }}$ & $19,4(106)^{\mathrm{abc}}$ & $25,8(104)^{\text {abcde }}$ & $27,3(110)^{\mathrm{abc}}$ \\
\hline TCL6 & $4,9(75)^{\mathrm{e}}$ & $7,7(118)^{\text {abcde }}$ & $12,9(70)^{\mathrm{f}}$ & $17,3(95)^{\text {abcdef }}$ & $17,8(72)^{\mathrm{de}}$ & $24,9(100)^{\text {abcde }}$ \\
\hline TB1 & $7,4(114)^{\text {abcde }}$ & $5,1(78)^{\mathrm{de}}$ & $18,3(100)^{\text {abcdef }}$ & $11,7(64)^{\mathrm{f}}$ & $25,7(104)^{\text {abcde }}$ & $16,9(74)^{e}$ \\
\hline TJ5 & $7,3(112)^{\text {abcde }}$ & $6,8(105)^{\text {bcde }}$ & $19,2(105)^{\text {abcdef }}$ & $17,8(97)^{\text {abcdef }}$ & $26,5(107)^{\text {abcde }}$ & $24,5(99)^{\text {abcde }}$ \\
\hline TCL4 & $6,8(105)^{\text {bcde }}$ & $7,2(111)^{\text {bcde }}$ & $14,3(78)^{\text {cdef }}$ & $16,5(90)^{\text {abcdef }}$ & $21(85)^{\text {bcde }}$ & $23,6(95)^{\text {abcde }}$ \\
\hline $\mathrm{TC} 2$ & $7,1(109)^{\text {bde }}$ & $5,7(88)^{\mathrm{de}}$ & $17,4(95)^{\text {abcdef }}$ & $13,9(76)^{\operatorname{def}}$ & $24,5(99)^{\text {abcde }}$ & $19,6(79)^{\mathrm{de}}$ \\
\hline TD6 & $7,1(109)^{\text {bcde }}$ & $5,7(88)^{\mathrm{de}}$ & $18,3(100)^{\text {abcdef }}$ & $15(82)^{\text {bcdef }}$ & $25,3(102)^{\text {abcde }}$ & $20,7(83)^{\text {cde }}$ \\
\hline TM1 & $5,9(91)^{\text {cde }}$ & $7,1(109)^{\text {bcde }}$ & $17,5(96)^{\text {abcdef }}$ & $18,1(99)^{\text {abcdef }}$ & $23,4(94)^{\text {abcde }}$ & $25,1(101)^{\text {abcde }}$ \\
\hline TD3 & $6,7(103)^{\text {bcde }}$ & $7(108)^{\text {bcde }}$ & $18,1(99)^{\text {abcdef }}$ & $17,2(94)^{\text {abcdef }}$ & $24,8(100)^{\text {abcde }}$ & $24,1(97)^{\text {abcde }}$ \\
\hline TC6 & $7(108)^{\text {bcde }}$ & $5(77)^{e}$ & $19(104)^{\text {abcdef }}$ & $13,7(75)^{\text {ef }}$ & $26(105)^{\text {abcde }}$ & $18,6(75)^{\mathrm{de}}$ \\
\hline ТР3 & $6(92)^{\text {cde }}$ & $6,8(105)^{\text {bcde }}$ & $13,6(74)^{\mathrm{f}}$ & $19,4(106)^{\text {abcd }}$ & $19,6(79)^{\text {de }}$ & $26,3(106)^{\text {abcde }}$ \\
\hline T. refer. & $6,7(103)^{\text {bcde }}$ & $6,3(97)^{\text {bcde }}$ & $13,7(75)^{\mathrm{ef}}$ & $14,8(81)^{\text {bcdef }}$ & $20,4(82)^{\text {cde }}$ & $21,4(86)^{\text {bcde }}$ \\
\hline T. abs. & $6,5(1$ & $100)^{\text {bcde }}$ & $18,3(10$ & $00)^{\text {abcdef }}$ & $24,8(1$ & 00) \\
\hline TV3 & $6,3(97)^{\text {bcde }}$ & $5,7(88)^{\mathrm{de}}$ & $14,7(80)^{\text {bcdef }}$ & $16,1(88)^{\text {abcdef }}$ & $20,9(84)^{\text {bcde }}$ & $21,9(88)^{\text {bcde }}$ \\
\hline TCL2 & $6(92)^{\text {cde }}$ & $5,9(91)^{\text {cde }}$ & $13,9(76)^{\text {def }}$ & $14,7(80)^{\text {bcdef }}$ & $19,8(80)^{\text {cde }}$ & $20,6(83)^{\text {cde }}$ \\
\hline TE5 & $5,8(89)^{\mathrm{cde}}$ & $5,1(78)^{e}$ & $15,2(83)^{\text {abcdef }}$ & $13(71)^{\mathrm{f}}$ & $21(85)^{\text {bcde }}$ & $18(73)^{\mathrm{de}}$ \\
\hline TV4 & $5,1(78)^{\mathrm{de}}$ & $5,4(83)^{\mathrm{de}}$ & $16,1(88)^{\text {abcdef }}$ & $14,9(81)^{\text {bcdef }}$ & $21,2(85)^{\text {bcde }}$ & $20,3(82)^{\text {cde }}$ \\
\hline
\end{tabular}

Letras distintas indican diferencias significativas $(\mathrm{p}<=0.05)$.

Los aislamientos endofíticos de Trichoderma spp., incrementaron los parámetros de peso con la interacción de los aislados dos y cuatro de Foc. Esto es favorecido por la colonización y el establecimiento de los aislamientos endofíticos de Trichoderma spp, en la rizosfera, promoviendo el crecimiento radical, del follaje y la disponibilidad de nutrientes como nitrógeno, fosforo, potasio, manganesio y hierro, vital para aumentar la resistencia a las enfermedades y mejorar la producción (Howell 2003; Vinale et al., 2008).

\section{CONCLUSIONES}

Veinte de cincuenta aislamientos endofíticos de Trichoderma spp., redujeron significativamente el crecimiento radial de los aislamientos de Fusarium oxysporum f. sp. cubense en comparación con los testigos referenciales de los aislados 2 y 4 de Foc.

Los hongos endofíticos TV3 y TB1 inhibieron desde un 38,82 a $53,46 \%$ de crecimiento radial de Fusarium oxysporum f. sp. cubense en comparación con los testigos referenciales de los aislados 2 y 4 de Foc. 
Los hongos endofíticos TB1, TC12, TC2, TC6, TC8, TC9, TCL1, TCL2, TCL4, TCL6, TD3, TD6, TD7, TE5, TJ5, TM1, TP3, TS2, TV3 y TV4 presentaron el mayor efecto de inhibición en la prueba de cocultivo y fueron seleccionados para la prueba de biocontrol.

Entre los aislamientos endofíticos de Trichoderma spp., seleccionados en prueba de antibiosis y prueba de biocontrol, no existió consistencia, por lo tanto la antibiosis como mecanismo de acción se descarta.

El aislado endofítico de Trichoderma spp., TJ5 retardó la aparición de los síntomas externos en más de tres semanas en comparación con los otros tratamientos, presentando $37,5 \%$ de incidencia.

Los hongos endofíticos TC9, TP3 y TCL1 redujeron desde un $92 \%$ hasta $90 \%$ la severidad de los síntomas externos de la enfermedad en comparación de los testigos referenciales.

Los hongos endofíticos TC9, TD3, TD6, TC12, TCL1, TC8 y TJ5 redujeron la severidad de los síntomas internos de la enfermedad desde un $74 \%$ hasta $63 \%$ en la decoloración del cormo en comparación a los demás tratamientos.

El hongo endofítico de Trichoderma spp., TCL1 incrementó el peso de la raíz, el peso del follaje y de la planta desde un 109\% hasta 148\% respectivamente en comparación al testigo absoluto.

Los aislados endofíticos de Trichoderma spp., TC9, TC8, TCL1 y TD6, presentaron los promedios más altos en el peso de la raíz con 0,67 y peso del follaje con 0,76 .

\section{AGRADECIMIENTO}

Al Servicio Alemán de Intercambio Académico (DAADCentroamerica), por el soporte financiero brindado para la realización de esta investigación y finalización de la Maestría de Agricultura Ecológica en el CATIE, Turrialba, Costa Rica. A la UNAN-León por mantenerme mi salario y puesto de trabajo durante los dos años de estudios en CATIE. Al personal de Docentes y trabajadores del CATIE y Biblioteca Orton. Al equipo científico de Bioversity internationalCATIE por su orientación en esta investigación.

\section{REFERENCIAS BIBLIOGRÁFICAS}

Aurore, G; Parfait, B; Fahrasmane, L. 2009. Bananas, raw material for making processed food product. Trend in Food Science \& Technology 20:78-91.

Cañizares, C. 2003. Estudio sobre poblaciones de hongos endofíticos provenientes de suelos supresivos al nematodo barrenador Radopholus similis (Cobb) Thorne en plantaciones comerciales de plátano en la zona de Talamanca, Tesis Mag. Sc. CATIE, Turrialba, CR. 2003, 75 p.

Di Rienzo, AJ; Macchiavelli, R; Casanoves, F. 2009. Modelos Mixtos en InfoStat. Tutorial, Grupo InFoStat, FCA, Universidad Nacional de Córdoba, AR. 193 p.

Harman, GE; Howell, CR; Viterbo, A; Chet, I; Lorito, M. 2004. Trichoderma species-opportunistic, avirulent plant symbionts. Nature Review Microbiology 2:43-56.

Howell, CR. 2003. Mechanisms employed by Trichoderma species in the biological control of plant diseases: The history and evolution of current concepts. Plant Disease 87(1):4-10.

Lara, D. 2009. Uso de bacterias endofíticas para el control biológico del Mal de Panamá (Fusarium oxysporum f. sp. cubense) en el cultivar Gros Michel (AAA). Tesis Mag. Sc. CATIE, Turrialba, CR. 85 p.

Menjivar, RD. 2005. Estudio del potencial antagonista de hongos endofíticos para el biocontrol del nematodo barrenador Radopholus similis en plantaciones de banano en Costa Rica. Tesis Mag. Sc. CATIE, Turrialba, CR. 69 p.

Mitov, N; Oliva, P. 1975. Estudio sobre el Mal de Panamá del Plátano en Cuba. Revista de Agricultura 8(2):12-29.

Moore, NY; Bentley, S; Pegg, KG; Jones, DR. 1995. Fusarium wilt of banana. INIBAP. Musa Disease no.5:1-4.

Orjeda, G. 1998. Evaluation of Musa germplasm for resistance to Sigatoka diseases and Fusarium wilt. INIBAP (International Network for improvement of banana and Plantain). Montepellier, Francia 19-29 p.

Pérez, L; Batlle, A; Chacón, J; Montenegro, V. 2009. Eficacia de Trichoderma harzianum A34 en el biocontrol de Fusarium oxysporum f. sp. cubense, agente causal de la marchitez por Fusarium o Mal de Panamá de los bananos en Cuba. Fitosanidad 13(4):259-263.

Pocasangre, LE. 2008. Estado actual y manejo del Mal de Panamá en América Latina y el Caribe. In XVIII Reunión internacional de la Asociación para la cooperación en investigaciones de bananos en el Caribe y la América Tropical. Acorbat 10 al 14 de nov. Guayaquil, EC. Resúmenes. p. 31.

Pocasangre, LE. 2009. Estado actual y manejo del manejo de Panamá en América Latina y el Caribe. In Reunión de grupo de interés sobre los riesgos de la raza tropical 4 de Fusarium, bbtv y otras plagas de Musáceas para la región del OIRSA, América Latina y el Caribe (Documentos de Programa y Resúmenes de la Reunión). 29 al 31 de julio. OIRSA, San Salvador, El Salvador. 18 p. 
Pocasangre, L; Pérez-Vicente, L. 2010. Impacto potencial de la entrada de la raza tropical 4 del Mal de Panamá (Fusarium oxysporum f. sp. cubense) en la industria bananera y platanera de América Latina y el Caribe. In Pocasangre, LE; Pérez, L; Martínez, E; Tapia, A; Guzmán, M; Brown, D. eds. Taller de entrenamiento sobre el diagnóstico y caracterización de la marchitez por Fusarium o Mal de Panamá. 22 al 26 de febrero, Turrialba, CR. 3 p.

Ploetz, RC. 1994. Panama disease: return of the first banana menace. International journal of pest management 40(4):326-336.

Ploetz, RC. 2006. Fusarium wilt of bananas is caused by several pathogens referred to as Fusarium oxysporum f. sp. cubense. Phytopathology 96(6):653-656.

Thangavelu, R; Velazhahan, R; Santhiamoorthy, S. 2003. Biocontrol of Fusarium wilt disease. In 2nd International symposium on fusarium wilt on banana. Programme and abstracts. 22-26 sept. Salvador de Bahia, BR. 34 p.

Vinale, F; Sivasithamparam, K; Ghisalberti, EL; Marra, R; Woo, S.L; Lorito, M. 2008. Trichoderma-plant-pathogen interactions. Soil Bioloy \& Biochemistry 40:1-10.

Woo, SL; Scala, F; Ruooco, M; Lorito, M. 2006. The molecular biology of the interactions between Trichoderma spp., Phytopathogenic Fungi, and plants. Phytopathology 96:181-185. 